\section{PWE-2 CLINICAL AND RADIOLOGICAL CHARACTERISATION OF POLYCYSTIC LIVER DISEASE}

Benjamin Giles*, Alison Dimmer, Zeshan Riaz, Joanna Dowman, Andrew Fowell, Richard Aspinall. Portsmouth Hospitals University NHS Trust, Portsmouth, UK

10.1136/gutjnl-2021-BSG.192

Introduction Patients with Polycystic Liver Disease (PLD) have a wide range of symptom severity and disease course but little is known about the clinical impact of PLD in the UK. The aim of our study was to examine the spectrum of PLD including severity, cyst phenotype, symptoms and treatment in a large, secondary care population over a 10 -year period.

Methods We systematically searched radiology reports from 2011-2020 at a major acute hospital serving a catchment population of 675,000. 'Polycystic' and 'multiple cysts' were used as search terms to identify patients, excluding those without liver or renal cysts. Electronic patient records were interrogated to collect demographics, clinical information and radiology. Images were reviewed to confirm numbers and sizes of cysts.

Results We identified 484 patients with multiple liver cysts; 211 with isolated PLD and 273 with both liver and kidney cysts. Of the radiology images available to view, 133/234 $(57 \%)$ met the accepted definition of PLD (>10 liver cysts). Overall, 39/484 (8\%) had symptoms from hepatic cysts whereas 26/133 (20\%) meeting the PLD definition were symptomatic with abdominal pain being the most common symptom (85\%). The mean age of first reporting PLD symptoms was 56 (range 36-84 years). Females were a higher proportion of both symptomatic patients $(72 \%)$ and those requiring treatment $(82 \%)$. Symptoms correlated with cyst number, occurring in $0-10$ cysts $6 / 101$ (6\%), 11-20 cysts $6 / 42$ (14\%), >20 cysts $20 / 91(22 \%)$ respectively. Cyst size similarly impacted symptoms and 218/229 (95\%) of patients with small $(<5 \mathrm{~cm})$ liver cysts were asymptomatic. The mean largest cyst size was 4.0 $\mathrm{cm}$ (range 0.3-14.8) in asymptomatic people and $8.6 \mathrm{~cm}$ (range 4.3-21.0) in those with symptoms. Only 16/484 (3\%) received targeted treatment for hepatic cysts with fenestration being the most common procedure performed, followed by resection and liver transplantation. No patients received somatostatin analogues during the study period.

Conclusions This is the largest systematic study of a PLD population from the UK and describes the clinical spectrum of disease. We found patients with multiple simple liver cysts rarely became symptomatic or required treatment. However, defined PLD was more severe in females with a higher rate of symptoms and need for specialist treatment. Symptomatic disease increased when multiple hepatic cysts exceeded 10 in number or grew larger than $5 \mathrm{~cm}$. Our data informs the natural history of PLD including the need to access appropriate care for symptomatic disease and highlights the importance of the recently established UK PLD Registry [https://clinicaltrials. gov/ct2/show/NCT04645251].

\section{PWE-3 UTILISING ILFT TO INCREASE THE IDENTIFICATION AND DIAGNOSIS OF HEPATITIS B AND C INFECTIONS}

${ }^{1}$ Callum Livingstone*, ${ }^{1}$ lain Macpherson, ${ }^{2}$ Jennifer Nobes, ${ }^{2}$ Elizabeth Furrie, ${ }^{1}$ Michael Miller, ${ }^{2}$ Ellie Dow, ${ }^{1}$ John Dillon. 'Gut Group, University Of Dundee, Dundee, UK; ${ }^{2}$ Department of Blood Sciences, NHS Tayside, Dundee, UK

10.1136/gutjnl-2021-BSG.193
Background Hepatitis B and C Virus (HBV and HCV) infections can lead to fibrosis, cirrhosis, and ultimately death; yet, $\mathrm{HBV}$ and HCV patients often go undiagnosed. Indeed, 50\% of UK HCV infections are estimated to be undiagnosed. These individuals likely being older and not having overt risks for HCV infection.

Automated testing of abnormal LFTs for HBV and HCV may help find these patients. Intelligent liver function testing (iLFT) is now routine in NHS Tayside and all abnormal LFTs are reflex tested for HBV and HCV. This may give additional opportunity for case finding in difficult to reach populations.

Methods Retrospective cohort analysis of patients who initiated iLFT testing, triggered at an ALT value above 30, from August 2018 to August 2020 and received an HBV or HCV outcome.

Results For HCV, 49 patients out of 6791 (0.7\%) were confirmed to be $\mathrm{HCV}$ antibody positive. 29 antibody positive patients (59\%) had detectable HCV RNA levels, predominantly Genotype 3 (34\%), suggestive of an active infection. 28/29 patients (97\%) commenced treatment on average 65 days from the iLFT request date (95\% CI 55.4 - 74.6 days) and 1 patient failed to attend.

26/28 patients (93\%) completed their treatment, 1 experienced side-effects and 1 was lost to follow-up. An intentionto-treat analysis demonstrated 21 patients $(75 \%)$ to have a sustained viral response (SVR). 19 SVR patients (90\%) were discharged or due for discharge and 2 continued under review due to the presence of fibrosis and/or cirrhosis.

Patients who underwent HCV treatment were on average 52-years-old, predominantly declared to having never injected drugs $(75 \%)$ and primarily resided in more affluent areas (75\%).

For HBV, iLFT identified 2 chronic active and 4 inactive carrier HBV infected patients. The 2 chronic active patients were initiated on treatment and all 6 patients remain under review. 1 chronic patient was also found to be Delta antigen positive.

The average age of the $6 \mathrm{HBV}$ patients was 52-years-old and all 6 patients (100\%) lived in more affluent areas.

Conclusions iLFT successfully identified patients with HBV and $\mathrm{HCV}$ infections and provided an opportunity for cure and/or continued follow-up. Of note, most patients treated for HCV gave no history of injecting drugs and resided in more affluent areas. NHS Tayside has already identified approximately 90\% of prevalent HCV cases. Therefore, iLFT has potential to be even more effective in areas with lower diagnosis rates and is a useful tool for identifying 'hard to reach' HCV positive patients.

\section{PWE-4 CLINIC NON-ATTENDANCE IN ALCOHOL-RELATED LIVER DISEASE IS VERY COMMON, AND LINKED TO POORER 1-YEAR OUTCOMES}

Neil Gordon*, Yuxiang Gao-Du, Amy Hicks, Lynsey Corless. Hull University Teaching Hospitals NHS Trust, Hull, UK

\subsection{6/gutjnl-2021-BSG.194}

Introduction Mortality following acute decompensation (AD) of alcohol-related chronic liver disease remains high despite significant advances in in-patient therapeutics and supportive care. Ongoing specialist support is recommended for all survivors and yet many patients are lost to follow up over time, 